\title{
Stratified Squamous Epithelium
}

National Cancer Institute

\section{Source}

National Cancer Institute. Stratified Squamous Epithelium. NCI Thesaurus. Code C13180.

Epithelium consisting of multiple layers of cells that are flattened and more cuboidal toward the base. Its function is protection. Outer layers may be dead and hardened

(keratinized) such as with skin or on top of wet, mucous surfaces such as the cornea 